\title{
Editorial Note: Design rules for modulation-doped AlAs quantum wells [Phys. Rev. Materials 1, 021002(R) (2017)]
}

\author{
Yoon Jang Chung, K. W. Baldwin, K. W. West, D. Kamburov, M. Shayegan, and L. N. Pfeiffer
} (Received 19 July 2021; published 27 July 2021)

DOI: 10.1103/PhysRevMaterials.5.079902

This paper was published on 17 July 2017 in the original form of submission, without paragraph breaks throughout the paper. To enhance the readability of the paper, we bring to your attention the updated arXiv version of the paper [1], which contains the missing paragraph breaks.

[1] Y. J. Chung, K. W. Baldwin, K. W. West, D. Kamburov, M. Shayegan, and L. N. Pfeiffer, arXiv:1707.09011v2 (2021). 